
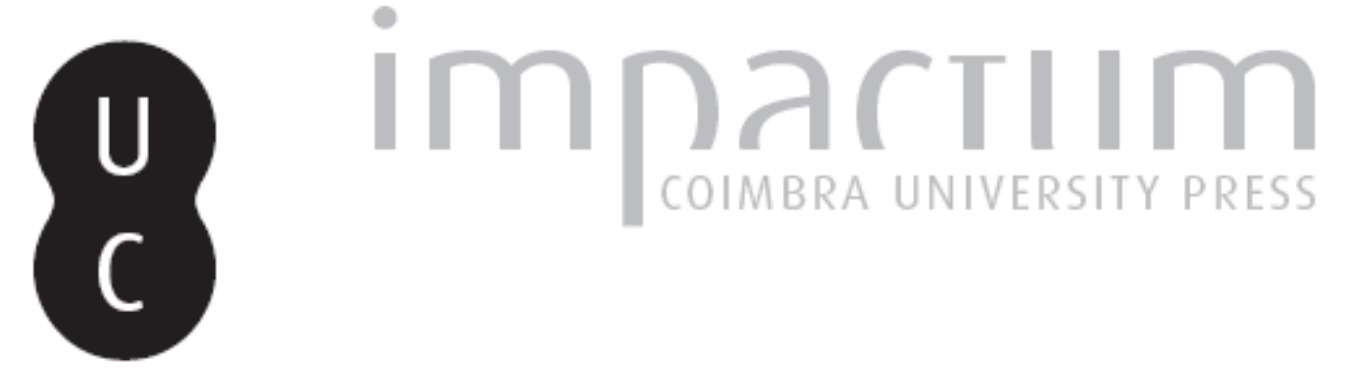

\title{
O conto dos chineses e i personaggi come "figure di funzione": una categoria vittoriniana nell’opera di José Cardoso Pires
}

\author{
Autor(es): $\quad$ Bettini, Clelia \\ Publicado por: Imprensa da Universidade de Coimbra \\ URL \\ persistente: \\ URI:http://hdl.handle.net/10316.2/42648 \\ DOI: \\ DOI:https://doi.org/10.14195/0870-8584_2_14
}

Accessed : $\quad$ 26-Apr-2023 12:32:44

A navegação consulta e descarregamento dos títulos inseridos nas Bibliotecas Digitais UC Digitalis, UC Pombalina e UC Impactum, pressupõem a aceitação plena e sem reservas dos Termos e Condições de Uso destas Bibliotecas Digitais, disponíveis em https://digitalis.uc.pt/pt-pt/termos.

Conforme exposto nos referidos Termos e Condições de Uso, o descarregamento de títulos de acesso restrito requer uma licença válida de autorização devendo o utilizador aceder ao(s) documento(s) a partir de um endereço de IP da instituição detentora da supramencionada licença.

Ao utilizador é apenas permitido o descarregamento para uso pessoal, pelo que o emprego do(s) título(s) descarregado(s) para outro fim, designadamente comercial, carece de autorização do respetivo autor ou editor da obra.

Na medida em que todas as obras da UC Digitalis se encontram protegidas pelo Código do Direito de Autor e Direitos Conexos e demais legislação aplicável, toda a cópia, parcial ou total, deste documento, nos casos em que é legalmente admitida, deverá conter ou fazer-se acompanhar por este aviso.

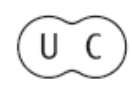




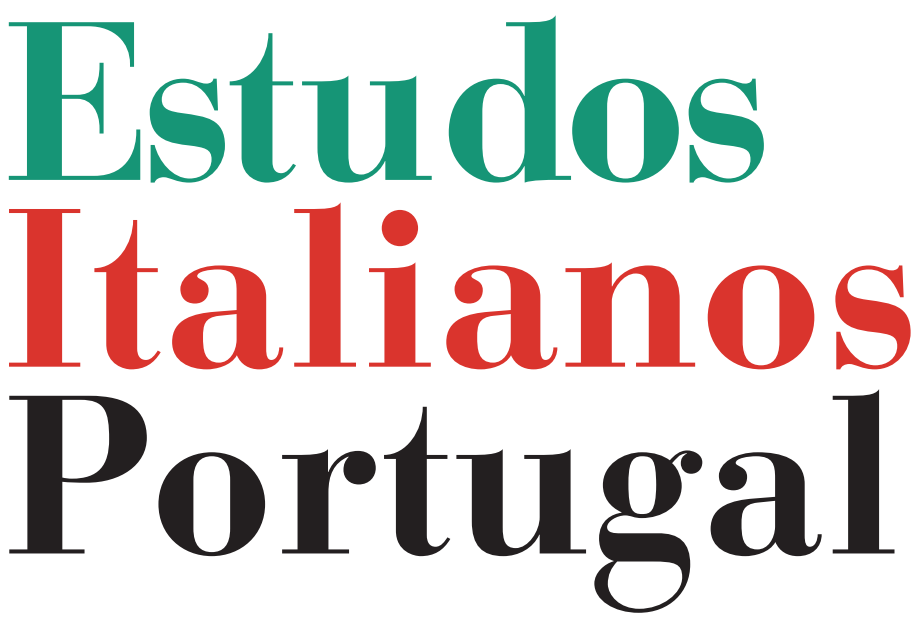

Instituto

Italiano

de Cultura

de Lisboa

Nova Série

$\mathbf{N}^{\mathbf{0}} 2$ 


\title{
O CONTO DOS CHINESES E I PERSONAGGI COME "FIGURE DI FUNZIONE". UNA CATEGORIA VITTORINIANA NELL'OPERA DI JOSÉ CARDOSO PIRES
}

\author{
Clelia Bettini ${ }^{\star}$
}

Argomento Di Questo Articolo sono due scrittori del '900: Elio Vittorini e José Cardoso Pires. I punti di contatto fra $\mathrm{i}$ due scrittori sono numerosi, ma in questa sede mi occuperò di un particolare modo di intendere e rappresentare il personaggio letterario che sembra transitare da Vittorini a José Carsdoso Pires: il personaggio come figura di funzione.

\section{Il personaggio figura di fUNZione SECONDO Elio VITTORINI}

In primo luogo è importante chiarire che cosa significa per Vittorini l'espressione figura di funzione applicata al personaggio. Lo spiega esemplarmente Maria Corti, a proposito di Conversazione in Sicilia, nel suo saggio che serve da introduzione al volume dei Meridiani dedicato a Elio Vittorini ${ }^{1}$ :

La novità strutturale di questo libro [Conversazione in Sicilia, n.d.a.] nato come seguito di Garofano Rosso e poi interrotto, sta nella sostituzione dei personaggi del realismo psicologico

* Clelia Bettini, nata a Livorno il 17-06-1978. Laureata in Letteratura Portoghese presso l'Università di Pisa, è stata borsista presso l'Università di Siena, dove ha appena concluso il Dottorato in Letteratura Comparata e Traduzione del Testo Letterario (2007). Ha tradotto dal portoghese i romanzi Equatore di Miguel Sousa Tavares (Premio Grinzane Cavour 2006) e Gente Felice con Lacrime, di João de Melo, entrambi per la Cavallo di Ferro Editore.

1 Corti, Maria, "Prefazione" a Vittorini: 1974, pp. X-LX. 
con quelli che Vittorini nelle Due Tensioni chiamerà le "figure di funzione", cioè personaggi portavoce cui pertiene natura di simboli più che di uomini².

Due sono i preziosi elementi interpretativi che Maria Corti ci fornisce per intraprendere un'indagine sul personaggio vittoriniano inteso come figura di funzione. Innanzitutto Corti "data" la sua comparsa nell'opera di Vittorini: Conversazione in Sicilia è il primo romanzo in cui troviamo questo tipo di personaggio. Il secondo elemento, conseguente e forse più importante, consiste nel definire come "novità strutturale" di Conversazione la soppressione del personaggio realistico in favore di una figura funzionale. È unanime il giudizio della critica nel ritenere Conversazione un romanzo di importanza capitale nel percorso letterario di Vittorini: scritto negli anni della Guerra di Spagna, segna il passaggio alla letteratura "impegnata" in senso politico-sociale, ed è specchio del costante interrogarsi sulla relazione fra scrittura e società, che lo accompagnerà sino agli scritti teorici dell'ultima fase della sua vita ${ }^{3}$. La teoria vittoriniana dei personaggi come figure di funzione assume dunque ancor più importanza quando la si ricollega, con Maria Corti, a Conversazione, definendola come novità strutturale su cui si fonda la differenza dell'opera da quelle che l'hanno preceduta.

La definizione vittoriniana di personaggio come figura di funzione è attestata per la prima volta, secondo Maria Corti, in Le due tensioni (1967). Prima ancora, la troviamo tuttavia in un'intervista rilasciata da Vittorini a Nello Ajello, pubblicata su L'Espresso del 10 maggio del 1964. Dice Vittorini:

Uno degli sforzi maggiori che deve compiere uno scrittore, oggi, è infatti di non produrre dei personaggi "privati" come si

${ }^{2} I b .$, p. XXVI.

${ }^{3}$ Si veda a questo proposito l'interessante ricostruzione e interpretazione di quegli anni di Anni Panicali, in Panicali: 1994, in special modo pp. 157-160. 
era avvezzi a fare da Balzac in poi, ma cercare di proiettarli al di là del verosimile, dotarli cioè di un significato culturale: farne delle "figure di funzione" come Don Chisciotte o Sancho Panza.

La riflessione sul personaggio - tanto nell'intervista citata, come negli appunti raccolti successivamente in Le due tensioni che sostano sulla scrivania di Vittorini dove siede Ajello mentre lo intervista - si inserisce nella più ampia riflessione vittoriniana sui "difetti" della letteratura realista, riflessione che occupa largo spazio fra gli scritti postumi dello scrittore siciliano. Vittorini è in pieno disaccordo con tale tipo di letteratura, in primo luogo perché essa offre "un'immagine totale e chiusa della realtà in modo tale da non lasciare margine a problemi né adito a dubbi, a interrogativi" ${ }^{4}$, ed anche perché, nel farlo, ricorre a un'argomentazione narrativa che Vittorini definisce "discorso autoritario". Il discorso "autoritario" è per Vittorini quello che crede e pretende di raffigurare la realtà così come essa effettivamente è, e lo scrittore che lo utilizza arriva a credersi "dio", l'autorità che tutto conosce e tutto controlla, in modo monolitico ${ }^{5}$. In un testo contenuto in Le due tensioni, dal titolo per noi certamente significativo, "Conv[ersazione in Sicilia]e dopo", Vittorini esplicita quale debba essere il vero interesse di un autore:

ciò che interessa all'autore non è una "mimesi" della realtà, (in cui si lascia alla realtà stessa, alla sua voce diretta, di "rendere" o "non rendere" presto o tardi come trasformazione di questa) ma un'utilizzazione della realtà che possa rendere immediatamente, subito, e costruire subito, per le forze storiche, un'arma, uno strumento di trasformazione, o insomma chiamata a trasformare ${ }^{6}$.

4 Vittorini: 1967, "realismo", p. 162.

5 Si vedano a questo proposito, oltre al già citato "realismo", gli scritti intitolati "i novecenteschi”, "naturalismo I", "realtà" fra gli altri, in Vittorini: 1967.

6 Ib., p. 67. 
Ed è in questo contesto teorico che si inserisce la riflessione vittoriniana sul personaggio ( e la sua definizione come figura di funzione) in un testo che porta il titolo di "concezione patrimoniale della letteratura e concezione operativa (scientifica)":

processo privatizzante (defunzionante) del personaggio - quanto era accessorio (pittoresco) nel personaggio fino al settecento incluso (tutto il suo aspetto privato, la sua carta di identità) diventa essenziale e anzi totale nel personaggio dell'ottocento.(ancora in Balzac il personaggio riesce ad essere in qualche modo figura di funzione - pur avendo accresciuto enormemente la sua parte di persona privata, per cui il père Grandet a tratti perde la sua funzione di avaro. ${ }^{7}$

\section{E ancora:}

il personaggio come figura di funzione e il personaggio (ottocentesco) come persona - (del tutto surrogato sociale - surrogato di creazione in senso teistico - surrogato in fondo biologico: surrogato che non cambia nulla né ha effetto storico alcuno $=$ tanto è vero che non ha saputo prevenire la storia, avvertirci sulla storia - ci ha dato un'idea tranquillizzante del piccolo borghese, non ha saputo veramente indicarci il pericolo insito nel piccolo-borghese che poi si è manifestato come fascista, come nazista -; qui se la letteratura fosse rimasta tale cioè con una funzione pubblica, e non si fosse privatizzata perdendosi dietro al sogno di creare persone, noi avremmo saputo in anticipo che cosa è veramente il piccolo-borghese, e avremmo avuto qualche possibilità di evitare il nazismo che ci ha rivelato, in contrasto appunto con le edulcorazioni idiote della letteratura, che cosa il piccolo-borghese sia, quale smania di torturatore, di sbirro, e di terrorista repressivo abbia in corpo $^{8}$

7 Ib., p. 96.

${ }^{8} \mathrm{Ib}$. 
Vittorini ritiene dunque che nel corso dell'ottocento sia avvenuta una progressiva "defunzionalizzazione" del personaggio, che ha portato a trasformare in essenziale tutto quanto era stato considerato accessorio sino a quel momento: il suo aspetto privato, la sua carta di identità. Per dirlo con la terminologia di Propp, nell'ottocento le parti variabili del personaggio, i suoi attributi divengono essenziali. Vittorini spiega la tendenza a creare personaggi persona in base alla teoria sull'“autoritarismo" della letteratura di matrice realista, e cala la propria riflessione teorica nel fluire della Storia, e non può prescindere dagli orrori di cui l'Europa è stata testimone. L'insistenza nel creare persone, e non personaggi, ha avuto, secondo Vittorini, effetti deleteri devastanti per chi guardi alla letteratura come a una forza dinamica che produce effetti e conseguenze nella realtà. Invece di far aprire gli occhi, di problematizzare una determinata componente della società novecentesca, la letteratura ha prodotto appena "edulcorazioni idiote" che hanno contribuito a nascondere la verità, anziché a cercarla e a comprenderla.

Partendo dal presupposto che il personaggio quale figura di funzione costituisca l'esatto contrario dell'ottocentesco personaggio persona, possiamo dedurne che le sue caratteristiche siano esattamente opposte a quelle di quest'ultimo. Il personaggio figura di funzione dovrà inoltre "cambiare qualcosa" ed "avere effetti di tipo storico": ciò che conta sono le sue azioni, le sue correlazioni con il sistema in cui è immerso. L'identità realistica dei personaggi è da considerarsi dunque accessoria, poiché svia, distrae, allontana il lettore da quella che è l'essenza del personaggio: la sua funzione. Deve perciò essere scabro, scarno, affinché la sua funzione dinamica si possa realizzare al massimo delle potenzialità. 
2. Il personaggio funZionale CONTRO LA PERSONA VIVA: UNA TEORIA EUROPEA

La categoria vittoriniana del personaggio come figura di funzione, che si allontana dal modello realista del personaggio inteso come rappresentazione mimetica di una persona viva, si inserisce in un'ampia corrente teorica che accompagna la riflessione sul personaggio sin dai primi decenni del novecento. La critica letteraria novecentesca ha visto nel personaggio l'elemento letterario che riflette maggiormente i cambiamenti della letteratura in sé, perché costituisce il più evidente fra i legami che uniscono l'uomo, la realtà e la sua rappresentazione letteraria. Il personaggio assume così un importante valore simbolico - più o meno consapevole - per chi produce letteratura e per chi se ne occupa. Un'unica linea di pensiero, che Arrigo Stara ha definito "linea dell'agire", lega fra loro le teorie di Jurij Tynjanov, illustre membro della scuola formalista russa, la Morfologia della Fiaba di Vladimir Ja. Propp, gli studi sulla mitologia germanica di Ferdinand de Saussure e lo strutturalismo degli anni '60, con Barthes, Greimas e Todorov. L'elemento centrale della "linea dell'agire" consiste nel considerare i personaggi letterari "non secondo ciò che essi sono, ma secondo ciò che essi fanno"10. Questa nuova teoria del personaggio, così come accade in Vittorini, si contrappone alla rappresentazione mimetica del personaggio così come la si conosce nella letteratura ottocentesca di matrice realista.

Nel 1924 Tynjanov affermava che la critica aveva ormai smesso di considerare i personaggi come persone vive, e ricorreva all'utilizzo del concetto di funzione:

9 Stara: 2004, pp. 175 ss.

${ }^{10}$ Barthes, Roland, Introduzione all'analisi strutturale del racconto, in A.A.V.V., L'analisi strutturale del racconto, p. 30. 
la correlazione di ciascun elemento dell'opera letteraria come sistema con gli altri e, di conseguenza, con tutto il sistema, io lo chiamo funzione costruttiva dell'elemento dato ${ }^{11}$

Il personaggio è un elemento del sistema opera letteraria, e come tale si pone in correlazione con gli altri: tale correlazione è per Tynjanov la funzione del personaggio. Il Nome, in Tynjanov rappresenta il personaggio, fa in modo che non lo si debba riferire continuamente definendolo, ne è simbolo. Tra il 1904 e il 1910, Saussure scriveva nei suoi appunti sulla mitologia germanica ${ }^{12}$ : "ciascuno dei personaggi è un simbolo di cui si può variare - esattamente come per la runa a) il nome, b) la posizione nei confronti degli altri, c) il carattere, d) la funzione, vale a dire le azioni". Saussure considera l'insieme delle leggende germaniche come un sistema simbolico chiuso, costituito da elementi che si relazionano reciprocamente, e i personaggi sono uno dei simboli inseriti nel sistema, secondo una visione molto simile a quella che sta alla base delle teorie sulla lingua che Saussure elaborerà pochi anni dopo. All'interno del sistema delle leggende germaniche, la funzione del personaggio corrisponde per Saussure alle azioni che questo compie. È nota inoltre l'importanza delle funzioni dei personaggi per la Morfologia della fiaba di Propp. Propp afferma:

11 Tynjanov, Jurij, L'evoluzione letteraria, in Todorov: 1965, pp. 126-143. Il passo citato si trova a p. 130.

12 Per una completa bibliografia sugli scritti relativi alla mitologia nordica di Saussure rimandiamo a un ampio e completo studio di Arrigo Stara (Stara: 2004 , p. 184, nota 92) sulla figura del personaggio letterario, cui questa nostra riflessione su una branca della teoria novecentesca del personaggio è profondamente debitrice, per la visione d'insieme, accurata e moderna, che fornisce del problema. Ci riferiamo in particolare alla terza parte del capitolo IV, intitolata La linea dell'agire, dove Stara documenta la linea di analisi e di interpretazione del personaggio letterario che si basa sull'importanza attribuita alle azioni. 
Cambiano i nomi (e con essi gli attributi) dei personaggi, ma non le loro azioni, o funzioni, donde la conclusione che la favola non di rado attribuisce un identico operato a personaggi diversi. Questo ci da la possibilità di studiare la fiaba secondo le funzioni dei personaggi. ${ }^{13}$

Anche per Propp funzione è un sinonimo di azione, e sullo studio delle funzioni dei personaggi basa l'intera sua teoria. La Morfologia di Propp e la straordinaria diffusione che essa ebbe in seguito alla prima traduzione inglese pubblicata nel 1958 - risonanza in gran parte dovuta alla polemica nata fra l'autore e Claude Lévi-Strauss - fu motore di riflessione per molti studiosi legati allo strutturalismo francese, che vi trovarono numerose consonanze con la propria riflessione sulla letteratura. Lévi-Strauss aveva infatti definito il proprio testo dedicato alla Morfologia di Propp, in una nota all'edizione italiana, come "un atto di riconoscimento verso una grande scoperta che doveva precedere di un quarto di secolo i tentativi compiuti da altri e dallo scrivente nella stessa direzione"14. Come abbiamo già accennato, nel 1966 esce un numero speciale della rivista Communications dedicato interamente alla analisi strutturale del racconto. Scrive Roland Barthes nell'Introduzione al volume:

L'analisi strutturale, molto attenta a non definire il personaggio in termine d'essenze psicologiche si è sforzata fino ad ora, attraverso ipotesi differenti, di cui si troverà l'eco in alcuni dei contributi che seguono, di definire il personaggio non come un "essere" ma come un "partecipante". ${ }^{15}$

L'analisi strutturale ignora l'aspetto psicologico del personaggio e si concentra sulle sue azioni, sul suo essere "par-

13 Propp: 1958, p. 26.

${ }^{14} \mathrm{Ib}$., p. 164.

${ }^{15}$ Barthes, Roland, cit., p. 30. 
tecipante" e non "essere". Su questa linea si collocano anche i contributi di Greimas, Todorov e Bremond pubblicati nello stesso volume: la teoria attanziale di Greimas insiste in particolar modo sulla centralità delle azioni dei personaggi, come spiega ancora Barthes:

A.J. Greimas ha proposto di descrivere e classificare i personaggi del racconto non secondo ciò che essi sono, ma secondo ciò che essi fanno (da qui il loro nome di attanti), nella misura in cui partecipano a tre grandi assi semantici che si trovano anche nella frase (soggetto, oggetto, complemento d'attribuzione, complementi circostanziali) perché sono la comunicazione, il desiderio (o la ricerca) e la prova. ${ }^{16}$

La categoria vittoriniana del personaggio come figura di funzione si inserisce dunque in una riflessione sul personaggio che percorre l'Europa dai primi anni del secolo ai fermenti e alle rivoluzioni degli anni' 60, partendo dal formalismo russo e dallo strutturalismo linguistico per arrivare allo strutturalismo letterario e alla semantica strutturale di Greimas. L'interesse di Vittorini per lo strutturalismo è del resto documentabile oggettivamente, oltre che deducibile dalle riflessioni sul personaggio oggetto di questa analisi. Nell'introduzione alla seconda edizione di Opera Aperta, Umberto Eco racconta le circostanze in cui Vittorini decise di pubblicare il suo saggio dal titolo "Del modo di formare come impegno sulla realtà" sul numero cinque de Il Menabò (1962), e nel farlo sottolinea il profondo spirito innovativo e il coraggio di un intellettuale affermato entro un determinato circolo di idee e amicizie, che decide di andare "controcorrente", attirandosi così gli odi e le accuse di una buona parte della critica italiana: 
Vittorini decise di affrontare il problema da un altro lato ${ }^{17}$ : come la situazione industriale influiva sui modi stessi della scrittura o se si vuole il problema dello sperimentalismo, ovvero letteratura e alienazione, o ancora come il linguaggio reagisce alla realtà capitalistica. Insomma, un bel nodo di problemi affrontati proprio nel modo che a quei tempi non piaceva alla sinistra "ufficiale", ancora crociana e neorealista (Come annotazione marginale, ci si vedeva con Vittorini quasi ogni sera nella libreria Aldrovandi, e Vittorini aveva sottobraccio la fonologia Trubetcoij: vaghi sentori strutturalistici nell'aria...) [...]Non fu un'operazione facile, non dico per me, ma per Vittorini, fu un atto di coraggio da parte sua, e tutti i vecchi amici lo accusavano di tradimento, anzi dovette scrivere alcune pagine di introduzione al numero in cui metteva le mani avanti (non ricordo se lui o Calvino parlarono poi ridendo di "cordone sanitario" indispensabilie). ${ }^{18}$

Se in base all'analisi del pensiero di Vittorini era possibile supporre ch'egli respirasse in quegli anni i "vaghi sentori strutturalistici" che aleggiavano anche sulla cultura italiana, il ricordo di Eco testimonia che nel 1962 l'autore di Conversazione in Sicilia, in una prospettiva assai più "concreta", "aveva sotto braccio" una delle opere più importanti dello strutturalismo linguistico, i Fondamenti di fonologia di Nijolaj Trubeckoj. Una nota apposta alla prima edizione italiana dell'opera di Trubeckoj, pubblicata dall'editore Einaudi nel 1971, giunge a ulteriore conferma del grande interesse di Vittorini per lo strutturalismo linguistico. Sul frontespizio del volume si legge infatti: 'L'edizione italiana di quest'opera è stata realizzata per consiglio di Elio Vittorini"19. Sebbene i Fondamenti siano stati pubblicati in lingua italiana solamente alcuni anni dopo la

${ }^{17}$ Eco si riferisce qui al rapporto fra industria e letteratura, che nel numero quarto de Il menabò, interamente dedicato al tema, era stato affrontato attraverso la visione che della vita industriale avevano alcuni narratori non sperimentali.

18 Eco: 1962, Introduzione alla $2^{\mathrm{a}}$ ed., p.VII.

19 Trubeckoj: 1971, p. IV. 
morte di Vittorini, il curatore e l'editore dell'opera ritennero necessario segnalare che il merito della "scoperta" dell'importanza dell'opera di Trubeckoj era da attribuirsi alla sempre viva curiosità intellettuale di Elio Vittorini.

\section{I PERSONAGgi FIGURE DI FUNZIONE: UNA CATEGORIA vittoriniana nell'opera di José Cardoso Pires}

José Cardoso Pires incontra per la prima volta Elio Vittorini nel 1958, a Milano, dove si era trasferito per lavorare qualche tempo al settimanale Epoca. Il periodo di "apprendistato" presso l'importante settimanale milanese doveva servire da presupposto a fondare una rivista simile in Portogallo, fortemente voluta dall'editore Figueiredo Magalhães, direttore della casa editrice di Lisbona Ulisseia, rivista che non vide mai la luce, perché bloccata dalla censura:

O Figueiredo Magalhães, editor da Ulisseia, queria fazer uma revista de grande qualidade. O Magalhães era um tipo muito culto, muito louco e com muito dinheiro. Depois acabou falido - com a nossa ajuda... Trazia o nome da revista, de que eu nunca gostei (A Semana). Queria uma coisa semanal, com piada. Então fui fazer um estágio numa grande revista da altura, da Mondadori, a Época, em Milão. E é em Milão que faço amizade com o Elio Vittorini. Mas o Magalhães, como era um gajo muito rico, estava convencido que a censura não existia. Quando apresenta a papelada para a revista semanal, aparece-lhe uma nega a dizer que a direcção - que era eu - não lhe merecia confiança, que sugerisse outros nomes. E o Magalhães teve a dignidade de não sugerir nada, pelo que a revista acabou sem ter começado. ${ }^{20}$

20 Entrevista a Inês Pedrosa, 24-06-1998, Pedrosa: I999; "Figueiredo Magalhães, direttore della casa editrice Ulisseia, voleva creare una rivista di grande qualità. Magalhães era un tipo molto colto, molto pazzo e con molti soldi. Finì per andare fallito - e anche grazie al nostro aiuto... Aveva già bell'e 
Durante l'estate di quello stesso 1958, in agosto, José Cardoso Pires trascorre parte delle vacanze estive a St. Raphael, e si reca quotidianamente alla vicina località di Plage de Salins, più esattamente a casa di Dyonis Mascolo e Marguerite Duras, per far visita proprio a Elio Vittorini, ospite della coppia francese. Di quell'estate ci restano una foto, un'intervista uscita sulla Gazeta Musical e de todas as Artes neanche un anno dopo - intervista mai ripubblicata - ed una rielaborazione successiva della stessa, in forma di saggio, composta a più riprese tra il 1963 e il 1976 e pubblicata nel 1977 nella raccolta di saggi E Agora José? ${ }^{21}$.

La foto che testimonia l'incontro dei due scrittori ritrae un Vittorini mediterraneo che si allunga su una sedia a sdraio al sole d'agosto, in costume e camicia, mentre un atlantico Cardoso Pires lo ascolta all'ombra, provvisto di calzoni, calze e scarpe, sotto una probabile tettoia, con una sigaretta fra le dita. Una bicicletta e arbusti bruciati dalla canicola fanno da sfondo alla scena. L'intervista che vi si affianca, pubblicata nel '59, rappresenta in sostanza il nucleo centrale delle riflessioni che Cardoso Pires svilupperà in seguito nel saggio-ricordo pubblicato quasi vent'anni dopo. Il legame fra i due testi - seppure così lontani nel tempo - è ben esplicitato dalla scelta del titolo: per l'intervista Conversando com o homem a propósito dos outros ${ }^{22}$, titolo leggermente modificato in Con-

pronto il nome della rivista, che a me non è mai piaciuto.(A Semana). Voleva fare un settimanale, interessante ed originale. Dunque me ne andai a lavorare come apprendista presso una grande rivista di allora, della Mondadori, Epoca, a Milano. Ed è a Milano che faccio amicizia con Elio Vittorini. Magalhães, però, siccome era uno straricco, era convinto che la censura non esistesse davvero. Al momento di consegnare tutte le scartoffie per partire con il settimanale gli arriva un parere negativo, perché la direzione della rivista - che ero io - non godeva della fiducia dell'ufficio, e che suggerisse altri nomi. Magalhães ebbe la dignità di non suggerire un bel niente, e così la rivista sfumò prima ancora di nascere".

21 Cf. Pires: 1977, pp. 55-93.

22 Cf. Pires: 1959. 
versações com o homem a propósito dos outros ${ }^{23}$, per il saggio del 1977. Un filo rosso vittoriniano attraversa il pensiero e l'opera di Cardoso Pires per almeno vent'anni della sua vita. Il titolo in questione allude a Os homens e os outros, titolo della traduzione portoghese di Uomini e no, pubblicata nel 1954, a sua volta calco esatto di quello della prima traduzione francese, pubblicata a Ginevra nel 1946, Les hommes e les autres ${ }^{24}$.

L'intervista del 1959, pubblicata a distanza ravvicinata dall'incontro dei due scrittori, costituisce un documento prezioso per comprendere le motivazioni del dialogo letterario che José Cardoso Pires intrattiene con Elio Vittorini. L'intervista è pubblicata in portoghese, ma si può supporre che i due scrittori dialogassero fra loro in francese, lingua che entrambi conoscevano, e probabilmente in italiano, visto che, come si è ricordato, José Cardoso Pires aveva trascorso alcuni mesi a Milano. Il testo si sviluppa lungo due filoni paralleli: Cardoso Pires conversa con il suo compagno di albergo, il fotografo americano dell'agenzia Magnum Photo, Bob Lammont Sterling, e con Elio Vittorini nella casa di Plage de Salins. Bob Sterling è un fotografo del new journalism, ritrae e documenta ogni genere di cose: guerre, matri-

23 Cf. Pires: 1977.

${ }^{24}$ Elio Vittorini si espresse duramente riguardo la scelta del titolo della prima edizione francese - pubblicata a Ginevra nel 1946 - in una lettera a Michel Arnaud, datata 7 luglio 1947, alla quale allega una breve nota, da pubblicarsi nella nuova edizione del romanzo che Gallimard sta preparando a Parigi: "Il titolo italiano di questo romanzo Uomini e no significa che noi, gli uomini, possiamo anche essere 'non uomini'. Mira cioè a ricordare che vi sono, nell'uomo, molte possibilità inumane. Ma non divide l'umanità in due parti: una delle quali sia tutta umana e l'altra tutta inumana. Il titolo francese Les hommes e les autres opera invece tale divisione, e disturba lo stesso contenuto del libro. Adottato in un'edizione svizzera che ha preceduto la presente ha però raggiunto notorietà fra $i$ critici e non si può più rifiutarlo. Viene dunque conservato per evitare che sorgano malintesi sull'identità dell'opera. Ma l'autore tiene ad avvertire che, pur suonando bene, è un titolo sbagliato", Vittorini: 1947a. p. 124. 
moni, un nero che si butta dal ponte di Brooklyn, un bambino che memorizza logaritmi. Si trova in Costa Azzurra per ritrarre la bella società europea: il pittore Bernard Buffet e Anita Ekberg, il " Circo Barnum da Inteligência do Século"25, perché anche gli intellettuali sono inclusi nel pacchetto Magnum, misurati in base alla loro "visibilità pubblica". La "cultura" da rotocalco serve da contrappunto ai dialoghi con Vittorini, lo scrittore della parola nitida che di primo mattino, in costume e scalzo, parla dell" "umiltà" dell' essere scrittore. La breve intervista serve a far conoscere brevemente Vittorini al lettore portoghese: vi si espongono gli ultimi progetti dello scrittore, le linee generali della sua idea di letteratura. Si citano molti estratti da Diario in Pubblico, libro che Cardoso Pires ha appena ricevuto in regalo dall'autore, uscito in libreria da pochi mesi. Si veda ad esempio il brano in cui Vittorini spiega che cosa significa per uno scrittore essere "umile": deve essere come suo padre, che quando faceva il maniscalco non dava ascolto a nessuno ma quando scriveva, allora sì, teneva di conto qualunque opinione gli si desse. Solo grazie a questo tipo di "umiltà" lo scrittore è ingrado di cercare la "verità", senza perdere di vista l'oggetto del suo scrivere ${ }^{26}$. Tuttavia, il terzo paragrafo dell'intervista del '59 è senza dubbio il più importante, ai

25 Pires: 1959 , p. 252 “ Circo Barnum dell'Intelligenza del Secolo”.

26 Si tratta del capitolo LXXXVIII di Uomini e no, uno dei capitoletti in corsivo, soppressi a partire dalla terza e definitiva edizione del romanzo. L'autore lo recupera però in Diario in Pubblico, con il titolo di "Autobiografia in tempo di guerra. Essere scrittore": "Io penso che sia molta umiltà essere scrittore. Lo vedo come fu in mio padre, che era maniscalco e scriveva tragedie, e non considerava il suo scrivere tragedie di più del suo ferrare cavalli. Anzi, quando era a ferrare cavalli, mai accettava che gli dicessero: 'non così, ma così. Tu hai sbagliato'. Guardava coi suoi occhi azzurri, e sorrideva o rideva; scuoteva il capo. Ma quando scriveva dava ragione ognuno per qualunque cosa.", Vittorini: 1957, pp. 191-195. È interessante notare che lo stesso brano sarà citato da JCP, in traduzione, anche nel saggio del 1976. 
fini di comprendere l'interesse di Cardoso Pires per Elio Vittorini. Si intitola semplicemente Milano come Lisbona... "Milano come Madrid"27 è il titolo di un breve brano della serie "Autobiografia in tempo di guerra", uscito nel dicembre del 1944 su Il Politecnico e ripubblicato da Vittorini in Diario in Pubblico: è a questo brano che Cardoso Pires fa esplicito riferimento intitolando un paragrafo della sua intervista "Milano come Lisbona". Prosegue nella sua descrizione di Vittorini, riconosce in lui "a fala pausada dos homens do Alentejo"28, e molto della sollecitudine tipica dei popoli iberici: prepara il caffé, passeggia con i suoi ospiti e parla del vivere quotidiano. Vittorini afferma, "Milão como Madrid. Como Barcelona. E como Lisboa, por que não?” e Cardoso Pires spiega, "um passado comum, a mesma experiência histórica, têm esse condão: aproximar os extranhos. É isso a madre do melhor entendimento" 29 .

In "Milano come Madrid" si racconta dei bombardamenti americani su Milano dell'agosto del '43. Molti antifascisti si trovavano allora detenuti nelle carceri milanesi in attesa di giudizio. Le carceri non erano provviste di rifugi anti-aerei, e i detenuti trascorrevano i terribili momenti dell'attacco alleato nelle celle, udendo gli schianti, senza capire che cosa stesse realmente accadendo. Vittorini sceglie il dialogo, domanda e risposta, come spesso ama fare, fra alcuni personaggi, per raccontare e spiegare quei terribili momenti, la confusione di non capire chi è che rischia di ucciderli: amici? nemici? nemici dei nostri nemici? Nulla sembra più avere un senso. Sono nemici, dunque sono i fascisti. Giungono alla conclusione di non trovarsi in realtà a Milano, ma a Madrid, in Spagna, e a Guernica, e a Santander. Sono dunque

27 Vittorini: 1957, pp. 196-203.

28 Pires: 1959, p. 252; "il parlare lento degli uomini dell'Alentejo".

29 Pires: 1959, p. 252; "Un comune passato, la stessa esperienza storica, possiedono un potere straordinario, quello di avvicinare chi non si conosce. Ed è questa la matrice della più profonda comprensione". 
spagnoli. E anche cinesi, si trovano a Shangai, e in Polonia. Si trovano "sotto le bombe dei fascisti in tutti quei posti". Essere vittime del fascismo accomuna il destino di tutti quegli uomini. L'indomani vengono "traslocati" presso un carcere fuori Milano. Attraversano la città in fiamme, ormai irriconoscibile, su una camionetta dei carabinieri. La trasfigurazione di luoghi un tempo noti e familiari suggerisce ancora la stessa identica riflessione del giorno precedente: come Madrid, "andando verso Boadilla un giorno di bombardamento dalle colline, [...] l'agosto del '36, il marzo del '37 [...]. O era lo stesso che Nanchino in Cina [...] il giorno che nel porto sbarcarono i fascisti giapponesi e mille giunche bruciavano sul fiume, diecimila case bruciavano lungo il fiume, centomila cinesi bruciavano anche nell'acqua del fiume sotto il ferro e il fuoco di seicento aerei" 30 .

Un comune passato e un'identica esperienza storica possiedono un potere straordinario: avvicinare chi non si conosce. Questa è l'idea di Cardoso Pires, che spiega così in che senso Milano è come Lisbona. Lo scrittore portoghese sente e stabilisce una profonda affinità con Vittorini e l'esperienza storica ch'egli rappresenta, e condivide e ammira il suo modo di intendere la letteratura. Il breve paragrafo Milano come Lisbona continua con alcune riflessioni private, alla sera, di nuovo in albergo: "A jovem literatura e a moralização da actividade artística a partir de 1945 em Itália. 'Sem jornais nem crítica aberta não podia haver novos escritores' - palavras de Vittorini. 'A reputação elaborava-se em ilhas privadas e não no diálogo da opinão pública' Milano como Lisboa..." 31 . Essere un giovane scrittore nell'Italia fasci-

30 Vittorini: 1957, p. 202.

31 Pires: 1959 , p. 253. "La letteratura giovane e la moralizzazione delle attività artistiche in Italia dopo il 1945, 'Senza giornali liberi e critica libera non potevano esistere nuovi scrittori' - parole di Vittorini. 'Ognuno si creava la propria reputazione all'interno di isole private e non nel dialogo dell'opinione pubblica.' Milano come Lisbona..." 
sta ed esserlo nel Portogallo del 1958 è dunque un'esperienza comune, una brutta esperienza comune.

Pochi mesi dopo l'incontro con Vittorini a Plage de Salins, Cardoso Pires scrive un breve racconto intitolato $O$ Conto dos Chineses, pubblicato esattamente vent'anni dopo in una sua antologia personale dal titolo O burro em pé. Uno studio comparativo di questo racconto e di Conversazione in Sicilia, che come si è detto è secondo Maria Corti il primo romanzo nel quale Vittorini impiega i personaggi quali figure di funzione, credo porti alla luce analogie sorprendenti tra due opere letterarie lontane sul piano geografico e linguistico, e anche su quello cronologico.

In primo luogo è immediato un riscontro di tipo tematico: l'intreccio narrativo del racconto di Cardoso Pires sembra costituire una diretta derivazione e una dilatazione del capitolo ventisette di Conversazione in Sicilia, interamente occupato da uno dei dialoghi fra il protagonista Silvestro e la madre, mentre questa compie il giro del paese per andare a fare le iniezioni agli ammalati di cui si occupa. Silvestro si interroga sul genere umano, e nel farlo si sofferma sulla figura di un cinese, come esempio, evidentemente, di uomo "diverso" dai siciliani che popolano la vita quotidiana della madre, ma la cui condizione sociale ed umana lo rende simile proprio a chi apparentemente si presenta come distinto, e al contempo obbliga a una riflessione che oltrepassi gli angusti confini regionali e nazionali. Quando Silvestro domanda alla madre se ha mai visto un cinese, la madre risponde: "Certo, $[\ldots]$ Ne ho visti due o tre... Passano per vendere le collane",32. E ancora Silvestro: "[il cinese] viaggia in mezzo a noi vendendo le sue collane e cravatte, con le sue cinture" 33 .

32 Vittorini: 1941, cap. XXVII.

$33 \mathrm{Ib}$. 
Conto dos Chineses è la storia di un muratore-contadino, guardiano di un capanno degli attrezzi, al quale si rivolgono due cinesi, che, viaggiando attraverso il Portogallo per vendere le loro mercanzie ed imbattutisi nell'uomo, decidono di fermarsi a chiedergli se ha del pane da vendere. Inizia a questo punto un lungo dialogo fra $i$ tre, in cui si confrontano usanze, abitudini e tradizioni, davanti a un piatto di minestra che il portoghese decide di offrire ai due cinesi. E anche questi cinesi sono "feirantes, destes que vendem carteirinhas lavradas e coisas de enfeitar raparigas" 34 .

Il personaggio del cinese, come esempio di alterità che può stimolare l'uomo a interrogarsi sul genere umano nella sua totalità, e favorire il lavoro di approssimazione alla verità, sembra transitare da Conversazione in Sicilia a Conto dos chineses, come veicolo di un messaggio storico-sociale. Il cinese è un miserabile fra i miserabili, sostiene Silvestro, "non ha cappotto, e ha il vestito stracciato e le scarpe rotte" e "e non ha pane, non vende mai nulla, non ha speranza. [...] più di tutti gli altri egli è senza speranza". Il guardiano portoghese si interroga invece su come "duas criaturas assim sugadas, duas almas sem pinga de gordura, não é verdade?, como podem eles, chineses, ter suor para deitar cá para fora?". Così magri, miserabili, non possono possedere neppure il sudore.

Proseguendo nell'elenco delle miserie del cinese, Silvestro invoca l'estraneità della lingua del paese ospitante come ulteriore prova della sua sventura: " Ma lui è un cinese, non conosce la nostra lingua e non può parlare con nessuno" 35 . Si noti la scelta, apparentemente ovvia, dell'aggettivo possessivo per caratterizzare la lingua sconosciuta al cinese. I due cinesi di Conto dos chineses parlano dapprima in una

34 Pires: 1979, p. 45; "venditori ambulanti, di quelli che vendono borsette ricamate ed altre chincaglierie per le ragazze".

35 Vittorini: 1941, cap. XXVII. 
lingua che "ninguém entendia", per poi in seguito domandare in portoghese al guardiano se ci fosse nei paraggi una taverna dove poter mangiare un boccone. Le due figlie del guardiano ridono dei cinesi, e allora uno di loro mormora la parola "meninas" ${ }^{36}$. Il narratore si affretta però a spiegare: "Claro que não dizia meninas como nós; dizia manine. Também não tinha dito ao chegar boa tarde. Tinha dito bôla tarde. E assim por diante" ${ }^{37}$.

Anche i cinesi di Conto dos chineses parlano male la lingua altra, non "como nós". Ritroviamo anche in questo secondo testo la marca distintiva della prima persona plurale per caratterizzare la lingua sconosciuta al cinese. Silvestro fornisce alla madre una descrizione fisica del cinese, descrizione che si basa essenzialmente nel sottolineare le differenze tra il tipo umano asiatico e quello caucasico: "Ma lui è cinese. Ha la faccia gialla, ha gli occhi obliqui, il naso schiacciato, gli zigomi sporgenti e forse fa puzza" 38 . Anche il guardiano di Conto dos chineses osserva l'aspetto dei suoi due ospiti cinesi, e lo fa seguendo una traccia molto simile a quella di Silvestro, anche se meno stilizzata:

Muito calados, os chineses comiam com uma velocidade danada. Toupeiras, ratos, bichos miúdos, era o que eles lembravam a mastigar. Mas só as bocas mexiam. [...] não devemos esquecer que a idade dos chineses engana muito. Aquela cara lisa, a barba que a bem dizer nem é barba é cabelo fraquito, verdadeira lã de rato, é que os faziam parecer mais novos ou melhor: sem idade ${ }^{39}$

36 Pires: 1979, p. 47.

$37 \mathrm{Ib}$.; "ovviamente quello non diceva bambine, come noi; diceva bimbane; e quando era arrivato non aveva detto buona sera. Aveva detto buola sela. E così via"

38 Vittorini: 1941, cap. XXVII.

39 Pires: 1979, p. 48; "Silenziosi, i cinesi mangiavano a una velocità spaventosa. Talpe, topi, piccoli animali, ecco cosa sembravano mentre masticavano. 
Esiste dunque fra i due testi esaminati un'evidente coincidenza tematica. Ciò che in Conversazione in Sicilia costituisce una breve porzione della lunga "conversazione" di Silvestro e degli altri personaggi, un confronto che non è un incontro reale, ma solo immaginato dal protagonista del romanzo, prende vita in Conto dos chineses, e si concretizza nella lunga serie di dialoghi fra i due personaggi cinesi e il portoghese. Se Silvestro interrogava la madre e si interrogava su chi fosse "più genere umano", se il povero cinese venditore ambulante o uno dei poveri siciliani cui la madre fa visita tutte le mattine, José Cardoso Pires fa condividere a un "povero portoghese" il proprio cibo con due venditori ambulanti cinesi, e dal loro incontro scaturiscono, attraverso - ancora una volta - una "conversazione", importanti considerazioni ${ }^{40}$. L'uomo, infatti, paragona costantemente se stesso e la propria cultura a quella dei suoi due ospiti. A un certo punto offre loro un pezzetto di formaggio di capra, ma i due non lo accettano. Allora l'uomo domanda loro se proprio non gli piace quel formaggio o se invece non lo conoscono, perché in Cina non si fabbrica. I due cinesi rispondo di sì, che in Cina si fa formaggio di latte vaccino e caprino, formaggio di ogni sorta. "Mesma coisa que aqui, patrão. Mesma coisa, mesma coisa" ${ }^{41}$, rispondono i due cinesi. E la "conversazione" prosegue ancora sul tema del cibo, in un botta e risposta scarno e serrato. Successivamente,

Ma erano solo le bocche a muoversi. [...] non dobbiamo dimenticare che l'età dei cinesi trae spesso in inganno. La pelle liscia, la barba che non è proprio barba, ma sono capelli debolissimi, una vera e propria lana di topo, ecco che cosa li fa sembrare più giovani o meglio: senza età".

40 Non riteniamo opportuno insistere sull'importanza del dialogo e dell'interrogazione priva di risposta nell'opera di Elio Vittorini, e soprattutto in Conversazione in Sicilia, più volte sottolineata dalla critica. Ci limitiamo a sottolineare ancora una coincidenza, questa volta sul piano stilistico, fra i due testi presi in esame.

41 Pires: 1979, p. 52; "Proprio come qui, padrone. Proprio come qui". 
poco prima di rimettersi in cammino, i due visitatori tracciano alcuni ideogrammi cinesi su un quaderno - si tratta di un modo di registrare le vendite - e ne spiegano il significato al guardiano, che chiede loro di scrivere parole a lui familiari come "uccellino" o "vino". I cinesi ripartono, e l'uomo non stacca gli occhi dal quaderno:

Lembrava-lhe a maneira como ele próprio, que não sabia escrever, apontava as suas coisas: un risco para cada saco de cimento, tantos quadrados para tantos milheiros de tijolo, uma cruz para as cargas de areia - e assim por diante. ${ }^{42}$

E conclude il guardiano, riflettendo sull'inatteso incontro di quel giorno: "Como nós [...] tal e qual como nós. No comer e em tudo"43.

Alcune significative somiglianze sul piano tematico, e il conseguente impiego del dialogo come mezzo espressivo necessario ai fini di chi scrive, evidenziano altre analogie fra i due testi: la presenza, per l'appunto, di personaggi figure di funzione nel testo di José Cardoso Pires, particolarmente evidenti nei due venditori ambulanti. E con questo torniamo al tema da cui questo articolo ha preso le mosse.

I nomi dei personaggi letterari raramente sono scelti a caso, ma quelli dei personaggi vittoriniani, che come lui stesso afferma necessitano di spersonalizzarsi, sono spesso costruiti prendendo una (e una sola) caratteristica del personaggio - non di rado una caratteristica fisica - e promuovendola, per così dire, di grado, attraverso l'uso delle lettera maiuscola. È il caso dei due personaggi che Silvestro incontra durante il viaggio in treno da Milano alla Sicilia, Coi

${ }^{42} \mathrm{Ib}$., p. 56; "Gli ricordavano il suo modo di appuntare le cose, lui che non sapeva scrivere: un trattino per ogni sacco di cemento, un quadrato ogni migliaio di mattoni, una croce per ogni carico di rena, e così via".

${ }^{43}$ Ib., p. 56; "Come noi [...] proprio come noi. In ogni cosa, anche nel mangiare". 
Baffi e Senza i Baffi, ma anche del Muso-di-Fumo di Il Sempione strizza l'occhio al Frejus, dove l'uso dei trattini sottolinea la trasformazione di una caratteristica fisica sintetizzata con più parole sintagma che diventa attraverso ciò che lo identifica il nome del personaggio.

Raramente però il personaggio figura di funzione è introdotto da Vittorini in medias res, privando il lettore di una breve spiegazione. Restiamo sui binari di Conversazione in Sicila, e esaminiamo il caso di due dei personaggi appena citati, Coi Baffi e Senza Baffi. Ecco l'incontro di Silvestro con questi due personaggi:

Erano due voci da sigaro, forti, e strascicate, dolci in dialetto. Parlavano siciliano, in dialetto.

Affacciai la testa sul corridoio e li vidi al finestrino, due uomini di persona massiccia, tarchiati, in cappello e cappotto, uno con i baffi, l'altro no, due siciliani di tipo carrettiere, ma ben messi, floridi, presuntuosi, nella nuca e la schiena, eppur con qualcosa di simulato e goffo che, forse, in fondo, era timidezza. ${ }^{44}$

Dopo aver detto che l'uno ha i baffi e l'altro non li ha, Silvestro passa a designarli come "Coi Baffi" e "Senza Baffi" - si noti l'uso della lettera maiuscola - mentre ascolta e riflette sul dialogo che intercorre fra i suoi due compagni di viaggio. Anche i cinesi di Cardoso Pires sono dotati di una breve presentazione. Quando si avvicinano al capanno dove sta di guardia il protagonista del racconto, l'uomo li sente, come abbiamo visto, discutere in una lingua incomprensibile e li descrive: "Pegaram-se em discussão, discussão mansa, conversa. Um tinha focinho de rato e dizia uma coisa, o outro tinha dentes de ouro e dizia outra" ${ }^{45}$.

44 Vittorini: 1941, cap. V.

45 Pires: 1979, p. 45; "Cominciarono a discutere. Era una discussione tranquilla, una conversazione. Uno aveva il muso di topo e diceva una cosa, l'altro i denti d'oro, e ne diceva un'altra". 
Subito dopo il narratore della vicenda prende a designarli "Focinho de Rato" e "Sorriso Dourado", secondo un procedimento esattamente sovrapponibile alla tecnica vittoriniana. Introduce i personaggi, ne evidenzia una caratteristica - fisica in questo caso - e la trasforma in nome proprio, con la maiuscola. I due cinesi possono essere inoltre considerati, a pieno titolo, due personaggi figure di funzione perché racchiudono in sé tutte le caratteristiche che abbiamo visto essere peculiari dell'idea di personaggio propria di Vittorini. Il nome, come si è detto, coincide perfettamente con la tipologia dei nomi scelti da Vittorini per i suoi personaggi. La descrizione e la caratterizzazione dei due personaggi è, inoltre, decisamente poco realistica: non ne fornisce una descrizione fisica esaustiva, ma per rapide pennellate successive, destinate a stilizzare più che uno statico elemento di una costruzione realistica, una figura in movimento. I cinesi non hanno un passato, ma hanno soltanto quello immaginato dall'uomo che incontrano. Il paese da cui provengono è rappresentato solo attraverso poche e scollegati immagini favolose che il guardiano portoghese ha costruito nella sua mente. Non hanno un futuro, per lo meno non ci è dato di conoscerlo con certezza. Di loro sappiamo solo che sono venditori ambulanti cinesi e che si stanno recando a Linda-a-Pastora, una località lontana da dove si trovano, dove si terrà una grande fiera. Il resto è solo una supposizione dell'uomo. I due personaggi si inseriscono dunque sulla scena, dentro la narrativa, e attraverso il dialogo, la "conversazione", la mettono in movimento. Sono privi di ciò che Vittorini considera "accessorio": non portano con sé alcun documento di identità.

In Conversazione in Sicilia per la prima volta nell'opera di Vittorini i personaggi sono intesi e creati come figure di funzione, e quest'innovazione costituisce secondo Maria Corti ciò che rende il romanzo diverso da tutti i prece- 
denti. Un modo di intendere il personaggio, come testimoniato dagli appunti di Le due tensioni e dall'intervista rilasciata al settimanale L'Espresso, che accompagnerà Vittorini fino all'ultima fase della sua vita di scrittore. Conto dos chineses è a sua volta il primo testo narrativo di Cardoso Pires dove i personaggi sono interpretabili come figure di funzione. Il procedimento creativo che sottende a nomi dei due venditori cinesi, e al modo in cui sono presentati al lettore, ci sembra una prova inconfutabile della legittimità con cui possiamo applicare loro a pieno titolo la definizione vittoriniana. L'evoluzione del discorso narrativo per dialoghi non fa che rafforzare l'idea di un legame fra il racconto di Cardoso Pires e Conversazione, così come la scelta dell'occasione narrativa: il venditore ambulante cinese come simbolo della miseria racchiusa in un involucro di totale alterità, che si rivela poi del tutto. Per un giovane autore portoghese che viveva e scriveva sotto la campana di vetro infrangibile del salazarismo, Elio Vittorini rappresentò senza dubbio uno spiraglio sulle più moderne tendenze della letteratura e della critica novecentesca, che nella spersonalizzazione del personaggio individuavano uno dei tratti più moderni e "sovversivi" della letteratura contemporanea. Il personaggio funzionale che cessa di essere antropomorfo non è un'invenzione esclusiva di Vittorini, ma le particolari caratteristiche della sua comparsa nell'opera di Cardoso Pires, e il dettaglio tecnico della costruzione del nome sono a mio avviso segni importanti della mutuazione diretta di un modo di intendere e rappresentare il personaggio letterario completamente diverso da quello previsto dal canone della letteratura di matrice realista che dominava la letteratura portoghese di quegli anni e le precedenti opere dello stesso Cardoso Pires.

L'incontro con Vittorini lascierà un'impronta concreta nell'opera di José Cardoso Pires. Molti dei personaggi che popolano i racconti e romanzi dell'autore de O Delfim 
- posteriori a Conto dos chineses - possono essere difatti interpretati come figure di funzione. Ne fornisco alcuni esempi. I soldati protagonisti di Carta a Garcia, racconto pubblicato nel 1963, hanno nomi come Rapa-Tacho (lett. Lecca-Pentola), Zabelinha (piccola Isabel, per il suo aspetto dolce, quasi effeminato), Espanhol (appassionato di coltelli, proveniente da una zona vicina alla frontiera spagnola) e Dois-Sessenta-e-Três (il numero che lo identifica come recluta) ${ }^{46}$. Il lettore non conosce il nome proprio di nessuno dei personaggi, identificati solo attraverso un nome che riflette una loro specifica caratteristica. Se alcuni sono veri e propri soprannomi, tipici di un contesto militare, non sarà inutile ricordare che il soprannome si adatta particolarmente al personaggio figura di funzione: rappresenta il personaggio attraverso una sua caratteristica e si sostituisce al nome proprio. Si pensi al caso di Enne 2 e dei personaggi di Uomini e no: il soprannome partigiano diventa il nome del personaggio figura di funzione. I nomi Rapa-Tacho e Dois-Sessenta-e-Três presentano inoltre la tipica costruzione vittoriniana delle parole maiuscole separate dai trattini ${ }^{47}$. Anche i personaggi di O Delfim, romanzo centrale per la comprensione dell'intera opera di José Cardoso Pires, possono essere letti come figure di funzione. Del protagonista conosciamo il nome, lo stesso dei dieci avi che lo hanno preceduto: Tomás Manuel da Palma Bravo. Tuttavia egli è quasi sempre chiamato Engenheiro dalla professione che non ha mai esercitato. Si pensi poi al vecchio venditore di biglietti della lotteria Velho-dum-Só-Dente - da notare l'uso della maiuscola e dei trattini - e alla femme fatale chiamata Dama das Unhas de Prata, i cui nomi sono entrambi scelti a partire da una caratteristica fisica estrapolata dal contesto generale e trasformata in simbolo per

46 Pires: 1963a, "Carta a Garcia".

47 Il soldato Dois-Sessenta-e-Três è ripreso inoltre successivamente nella figura del soldato Três-Dezasseis nel romanzo O Hóspede de Job. 
identificare il personaggio ${ }^{48}$. In un breve racconto del 1978 compare infine un bambino dai capelli rossi chiamato Ruivo-Estás-a-Arder (lett. Rosso-Stai-Bruciando, ovvero Rosso-Sei-nei-Guai, analogia fra il colore dei capelli e il fuoco che simboleggia un momento critico). E ancora Elias Santana, il poliziotto che indaga su un torbido crimine a sfondo politico in A Balada da praia dos cães è chiamato Covas (ovvero Fosse, per la sua vicinanza ai morti) o Elias Chefe (Elias Capo), mentre il maggiore assassinato Luís Dantas Castro diviene successivamente Dantas C.

Per concludere, possiamo notare che il personaggio figura di funzione si integra perfettamente nella concezione della letteratura di Cardoso Pires. Egli costruisce, infatti, realtà fortemente simboliche, quasi allegoriche, che proprio per questo assumono valore universale. Cardoso Pires accoglie dunque la lezione vittoriniana perché essa collima perfettamente con il suo modo di intendere la letteratura. Tuttavia egli non si limita ad imitare, semplicemente, un tratto stilistico dello scrittore siciliano, ma, come abbiamo visto, se ne appropria e lo rielabora al punto da trasformarlo in uno dei punti chiave del suo fare letteratura.

\section{BibLIOGRAFIA}

AA.VV., L'analisi del racconto, Bompiani, 1969. Traduzione italiana del numero 8 della rivista Communications, Seuil, 1966.

Eco, Umberto: $1962=$ Opera Aperta, $1^{a}$ ed. 1962, Bompiani. Si è consultata la $2^{\mathrm{a}}$ ed., Bompiani, 1995.

48 Pires: 1968; lett. l'Ingegnere, Vecchio-con-un-dente-solo, Dama dalle Unghie d'Argento. In O Delfim si considerino anche il personaggio del Batedor (colui che apre le battute di caccia) e del Regedor (l'autorità locale), la Dona da Pensão (la proprietaria della pensione), il Padre Novo (prete giovane). Si tratta infatti sempre di nomi non realistici, che fanno riferimento a un ruolo o a una professione e presentati con lettera maiuscola. 
O conto dos chineses e i personaggi come "figure di funzione" 303

Panicali, Anna: 1994 = Elio Vittorini, Mursia, 1994

Pedrosa, Inês: 1999 = José Cardoso Pires - Fotobiografia, D. Quixote, 1999.

Pires, José Cardoso

1959 = "Conversando com o Homem a propósito dos outros", in Gazeta musical e de todas as artes, Lisboa, 96, Março 1959, pp. 252-253.

1963, O Hóspede de Job, Arcádia, 1963

$1963 \mathrm{a}=$ Jogos de Azar, Arcádia, 1963.

1968 = O Delfim, Moraes, 1968.

1977 = E Agora, José?, Moraes, 1977.

1979 = O Burro-em-pé, Moraes, 1979.

Stara, Arrigo: $2004=$ L'Avventura del personaggio, Le Monnier Università, 2004.

Todorov, Tzvetan: 1965 = I formalisti russi, a cura di, Einaudi, 1968.

Trubeckoj, Nicolai S.: 1971 = Fondamenti di fonologia, Einaudi, 1971.

Vittorini, Elio: 1941 = Conversazione in Sicilia, Parenti, 1941.

1947a= Gli anni del Politecnico, Einaudi, 1977.

1957 = Diario in Pubblico, Bompiani, 1957

$1967=$ Le due tensioni. Appunti per un'ideologia della letteratura, a cura di Dante Isella, Il Saggiatore 1967.

$1974=$ Le opere narrative, a cura di Maria Corti, Mondadori, 1974. 